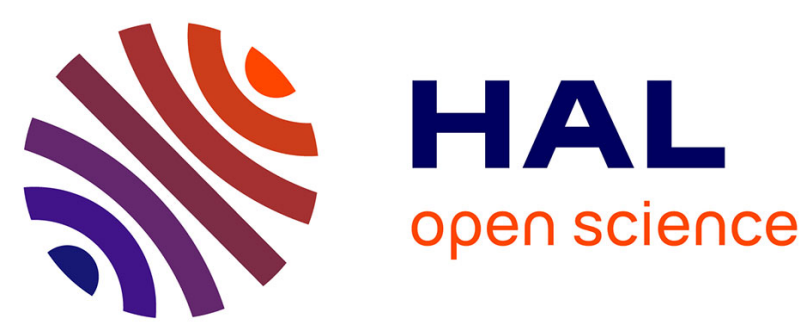

\title{
Anatomical predictors of cognitive decline after subthalamic stimulation in Parkinson's disease
}

Vincent Planche, Fanny Munsch, Bruno Pereira, Emmanuel de Schlichting,

Tiphaine Vidal, Jerome Coste, Dominique Morand, Ingrid de Chazeron, Philippe Derost, Bérangère Debilly, et al.

\section{To cite this version:}

Vincent Planche, Fanny Munsch, Bruno Pereira, Emmanuel de Schlichting, Tiphaine Vidal, et al.. Anatomical predictors of cognitive decline after subthalamic stimulation in Parkinson's disease. Brain Structure and Function, 2018, 223 (7), pp.3063-3072. 10.1007/s00429-018-1677-2 . hal-01814807

\section{HAL Id: hal-01814807 https://hal.uca.fr/hal-01814807}

Submitted on 24 Oct 2018

HAL is a multi-disciplinary open access archive for the deposit and dissemination of scientific research documents, whether they are published or not. The documents may come from teaching and research institutions in France or abroad, or from public or private research centers.
L'archive ouverte pluridisciplinaire HAL, est destinée au dépôt et à la diffusion de documents scientifiques de niveau recherche, publiés ou non, émanant des établissements d'enseignement et de recherche français ou étrangers, des laboratoires publics ou privés. 


\title{
Anatomical predictors of cognitive decline after subthalamic stimulation in Parkinson's disease
}

\author{
Vincent Planche ${ }^{1,{ }^{*}}$. Fanny Munsch ${ }^{2} \cdot$ Bruno Pereira $^{3} \cdot$ Emmanuel de Schlichting $^{4}$. \\ Tiphaine Vidal $^{5}$. Jerome Coste ${ }^{4}$. Dominique Morand ${ }^{3}$. Ingrid de Chazeron ${ }^{6}$. Philippe \\ Derost $^{1}$. Bérangère Debilly ${ }^{1}$. Pierre-Michel Llorca ${ }^{6}$. Jean-Jacques Lemaire ${ }^{4}$. Ana \\ Marques $^{1}$. Franck Durif ${ }^{1}$
}

1. Service de Neurologie, CHU Clermont-Ferrand, Université Clermont Auvergne, 58 rue Montalembert, 63000 Clermont-Ferrand, France

2. Service de Neuroradiologie diagnostique et thérapeutique, $\mathrm{CHU}$ Bordeaux, Université Bordeaux, 33000 Bordeaux, France

3. Unité de Biostatistiques, Direction à la Recherche Clinique et à I'Innovation (DRCl), CHU Clermont-Ferrand, 63000 Clermont-Ferrand, France

4. Service de Neurochirurgie, CHU Clermont-Ferrand, Centre National de la Recherche Scientifique (CNRS), Université Clermont Auvergne, 63000 Clermont-Ferrand, France

5. Centre Mémoire de Ressources et de Recherche (CMRR), CHU Clermont-Ferrand, 63000 Clermont-Ferrand, France

6. Centre Médico-Psychologique B (CMP-B), CHU Clermont-Ferrand, Université Clermont Auvergne, 63000 Clermont-Ferrand, France

Auteur correspondant : Vincent Planche planche.vincent@gmail.com

\begin{abstract}
We investigated whether pre-operative MRI measures of focal brain atrophy could predict cognitive decline occurring after deep brain stimulation (DBS) of the subthalamic nucleus (STN) in patients with Parkinson's disease (PD). For that purpose, we prospectively collected data of 42 consecutive patients with PD who underwent bilateral STN-DBS. Normalized brain structure volumes and cortical thicknesses were measured on pre-operative T1-weighted MRI. Patients were tested for their cognitive performances before surgery and 1 year after. After controlling for age, gender, pre-operative disease severity, change in dopaminomimetic dose after surgery and contact location, we found correlations: (1) between the variation of the total Mattis dementia rating scale (MDRS) score and left lateral ventricle volume $(p=0.032),(2)$ between the variation of the initiation/perseveration subscore of the MDRS and the left nucleus accumbens volume $(p=0.042)$ and the left lateral ventricle volume $(p=0.017)$ and $(3)$ between the variation of the backward digit-span task and the right and left superior frontal gyrus thickness ( $p=0.004$ and $p=0.007$, respectively). Left nucleus accumbens atrophy was associated with decline in the initiation/perseveration subscore with the largest effect size $(d=$ - 1.64). Pre-operative left nucleus accumbens volume strongly predicted postoperative decline in the initiation/attention subscore (AUC $=0.92, p<0.001,96.3 \%$ sensitivity, $80.0 \%$ specificity, 92.9\% PPV and 92.9\% NPV). We conclude that the morphometric measures of brain atrophy usually associated with cognitive impairment in PD can also explain or predict a part of cognitive decline after bilateral STN-DBS. In particular, the left accumbens nucleus volume could be considered as a promising marker for guiding surgical decisions.
\end{abstract}

\section{Keywords}

Parkinson's disease - Deep-brain stimulation - Subthalamic nucleus · Nucleus accumbens · MRI · Cognition 


\section{Introduction}

Bilateral deep brain stimulation (DBS) of the subthalamic nucleus (STN) is an effective treatment in advanced Parkinson's disease (PD) (Krack et al. 2003). However, postoperative decrease in cognitive functions has been described in short-term and long-term follow-up studies (Saint-Cyr et al. 2000; Alegret et al. 2001; Parsons et al. 2006; Contarino et al. 2007; Kim et al. 2014). It has been confirmed by a randomized clinical trial demonstrating that cognitive decline 6-months after surgery mainly concerns executive functions and especially verbal fluencies, and is not only due to disease progression but also to STN-DBS itself (Witt et al. 2008).

In order to select patients for surgery, several clinical predictive factors (age, disease duration, axial symptoms, and levodopa responsiveness) of the best post-operative outcomes were identified (Charles et al. 2002; Welter et al. 2002). However, they are poor predictors of cognitive decline after subthalamic stimulation in such selected and homogenous populations of patients with PD (Parsons et al. 2006; Daniels et al. 2010; Smeding et al. 2011; Welter et al. 2014). The localization of effective contacts within the STN and the lesional effect of the electrode trajectory are known to durably affect cognitive outcomes but they represent unpredictable therapeutic risks (Witt et al. 2013; Welter et al. 2014; Le Goff et al. 2015). Thus, there is a need to find new predictive biomarkers to refine the selection criteria of patients with PD undergoing subthalamic stimulation and to minimize cognitive side-effects.

Cognitive impairment in PD has been associated with cholinergic deficit or with cortical and limbic Lewy bodies (Emre 2004) and some longitudinal studies have identified the cortical and subcortical area of atrophy capable of predicting cognitive impairment over time, mostly the frontal and temporoparietal cortices, but also the hippocampus, the amygdala and the nucleus accumbens (Ibarretxe-Bilbao et al. 2012; Hanganu et al. 2014). However, there is to date no MRI parameter known to predict cognitive decline specifically following STN-DBS.

In this study, we hypothesized that the structural brain damage responsible for cognitive decline after STN-DBS is already present before surgery and, consequently, that pre-operative MRI biomarkers could explain postoperative decrease in cognitive functions. We then investigated whether the pre-operative atrophy of cortical or subcortical structures relevant for cognition in PD predicted cognitive decline after STN-DBS.

\section{Materials and methods}

Patients with PD

Forty-two consecutive patients that underwent bilateral STN-DBS in our PD center were prospectively included in this study. The patients were diagnosed according to the UK Parkinson Disease Brain Bank criteria (Hughes et al. 1992) and suffered from severe motor fluctuations despite optimal medical therapy. They were selected for surgery according to the following criteria (Charles et al. 2002; Welter et al. 2002): at least 50\% response to acute LDopa challenge, no postural instability during the best on period, absence of cognitive impairment (MMSE $\geq 25$ and Mattis Dementia Rating Scale (MDRS) $\geq 129$ ) and age $<70$ years. We collected clinical and neuropsychological data together with brain MRI within 1 month before surgery and the patients were followed up 1 year after bilateral STN-DBS for a new clinical and neuropsychological assessment.

The study was approved by the local institutional ethics review board (2017/CE 02). All the patients gave their written informed consent prior to surgery. 


\section{Surgical procedure and contact location definition}

The surgical procedure for bilateral STN implantation was carried out according to preoperative magnetic resonance imaging (MRI), anatomic mapping, intraoperative microrecordings and clinical assessment, as reported previously (Lemaire et al. 2007). The final effective contact location was identified 1-year after implantation on postoperative CT scans co-registered with pre-operative MRI (Lemaire et al. 2016) and determined regarding the compromise between substantial clinical improvement, adverse effects and optimized dopatherapy. As the anterior/ventral location of DBS contact (within the associative or limbic part of STN) is known to impair cognitive functions (Welter et al. 2014; Tsai et al. 2007), we assessed the contact location mainly in the antero-posterior axis. Then, the main axis of STN was subdivided into four longitudinal antero-posterior subdivisions [as previously described (Lemaire et al. 2016)]. We identified patients with an "anterior" location of contact if at least one effective contact on one side was situated in the most anterior quarter of the STN. In addition, we defined the contact location as "in", "out" ("in/out" in case of strong asymmetry) or at the superior border of STN ("border") as described previously (de Chazeron et al. 2016).

\section{Clinical and neuropsychological assessments}

Clinical and neuropsychological tests were performed 1 month before surgery and 1 year after. Parkinsonian symptoms were assessed with the modified Hoehn and Yahr scale and the Unified Parkinson's Disease Rating Scale (UPDRS). Before surgery, the patients were tested for motor functions with medication ("on") and after 12-h withdrawal of antiparkinsonian drugs (medication "off"). One year after STNDBS, the patients were tested in three conditions: "medication off/stimulation off", "medication off/stimulation on", "medication on/stimulation on". Cumulative L-Dopa equivalent daily dose (LEDD) was calculated according to usual conversion factors (Thobois 2006).

The neuropsychological evaluation was performed within 1 month before STN-DBS ("medication on") and 1 year after ("medication on/stimulation on") with the Mini Mental State Examination (MMSE), the Frontal Assessment Battery at Bedside (FABB), the forward and backward digit-span task (WAIS-III) and the Mattis Dementia Rating Scale (MDRS). The MDRS is one of the most commonly used scales in patients with parkinsonism in both clinical and research purposes (Dujardin et al. 2006; Marson et al. 1997) and has been previously used in studies about STN-DBS (Krack et al. 2003; Welter et al. 2014). MDRS subscores are known to be valid measures of their respective constructs: attention, initiation/perseveration, construction, conceptualization and memory (Marson et al. 1997). MDRS is very sensitive to executive dysfunction, especially the initiation/perseveration subscore, mainly driven by verbal fluency, which are frequently impaired after STN-DBS (Witt et al. 2008). Apathy and depression were assessed with the corresponding items of the UPDRS part I.

\section{MRI acquisition and post-processing}

The patients were scanned before STN-DBS on a 1.5T Siemens Avanto MRI system with an Elekta head coil. The pre-operative MRI protocol included specific sequences for STN localization and the following 3D T1-weighted sequence was used for volumetric analyses: $\mathrm{TR} / \mathrm{TE} / \mathrm{TI}=1870 \mathrm{~ms} / 2.97 \mathrm{~ms} / 1100 \mathrm{~ms}$, resolution $0.625 \times 0.625 \times 1.3 \mathrm{~mm}, 256 \times 228 \mathrm{~mm}$ FOV. For the volumetric analyses of subcortical (and hippocampal) structures, T1-weighted images were processed using the VolBrain system (http://volbr ain.upv.es) (Manjón and Coupé 2016). Volbrain was chosen to segment deep gray matter structures because it is a more accurate automatic segmentation software than other publicly available tools for these structures (Næss-Schmidt et al. 2016). To control for variations in head size, all volumes were scaled using the intracranial cavity volume of each patient. Cortical reconstruction of MRI data was performed using Freesurfer 5.1 (https ://surfe r.nmr.mgh.harva rd.edu) to study cortical 
thickness, as in previous longitudinal works studying cognition in PD (Mak et al. 2015; Segura et al. 2014). The pipeline was applied to all the T1-weighted MRI images as described previously (Fischl and Dale 2000) and included intensity normalization, removal of nonbrain tissue, segmentation, surface inflation, and topological correction. Automated cortical parcellation was performed according to Desikan atlas (Desikan et al. 2006) and the cortical thickness was measured as the closest distance from the gray/white matter boundaries to the gray/ CSF boundaries. In this exploratory study, 18 right and left cortical and subcortical areas relevant for cognition in PD were analyzed i.e. from the frontal to the temporoparietal cortex (excluding motor areas), together with basal ganglia and hippocampi (Mak et al. 2015; Hanganu et al. 2014; Segura et al. 2014; Camicioli et al. 2011).

\section{Statistical analyses}

Statistical analyses were performed with Stata software (version 12, StataCorp). The distribution of all continuous data were tested with the Shapiro-Wilk test. We compared presurgical to 1-year postoperative clinical and neuropsychological characteristics using Wilcoxon tests as the conditions for parametric tests were not met. Relationships between the variation of neuropsychological scores and pre-operative quantitative imaging variables were assessed using Spearman's correlation coefficients (according to statistical distribution) and represented graphically with a color-coded heatmap. Regarding the number of anatomical structures measured in this study ( $n=18$, right and left), we considered the issue of multiple comparisons using a Bonferroniadjusted significance threshold of $p<0.0014$. We further analyzed only potentially relevant findings [i.e., with at least a coefficient $|r \geq 0.3|$ in the correlation analysis, according to several recommendations (Altman 1990)]. Since cognitive decline after STN-DBS is small-to-medium according to Cohen's effect size $(-0.5<d<-0.2)$ (Witt et al. 2008), we defined patients with postoperative cognitive decline as patients with at least small $(d \leq-0.2)$ decreased performance compared to pre-operative scores. Patients with a more substantial cognitive decline [i.e., a decreased performance of at least - 1SD ( $d \leq-0.5)$ ] were also considered for sensitivity analyses. The effect size of brain atrophy in patients with cognitive decline compared to stable (or improved) patients was calculated (Cohen's $d$ ) and represented graphically with forest plots. Multivariate analyses were performed using multiple linear or logistic regression models to take into account adjustment on covariables fixed according to univariate results and clinical relevance: age, gender, disease severity (pre-operative Hoehn and Yahr stage), change in LEDD after surgery and the "anterior" position of the DBS contacts. These regression analyses were also used to evaluate the proportion of the variance of cognitive decline that could be explained by baseline factors. Lastly, receiver operating characteristic (ROC) analysis was performed to test the value of relevant MRI measures to predict patients with cognitive decline 1-year after DBSSTN compared to pre-operative scores [according to Youden recommendations (Altman 1990)]. All tests were two-tailed, with a type I error set at $\alpha=0.05$.

\section{Results}

\section{Demographics and pre- and post-operative assessments of patients}

The demographic characteristics of the 42 consecutive patients included in this study are summarized in Table 1. As expected, bilateral STN-DBS led to a significant reduction of total medical treatment (LEDD, $p<0.001$ ), together with a decrease of motor complication scores (UPDRSIV, $p<0.001$ ). The MDRS score tended to be lower 1 year after bilateral STN-DBS $(p=0.076, d=-0.37)$, driven by a significant decrease in its initiation/perseveration subscore $(p=0.003, d=-0.56)$. We also found a significant decrease in the backward digit-span task, $(p=0.007, d=-0.42)$ and in the MMSE score $(p=0.002, d=-0.43)$ (Table 1). 
Planche et al., 2018

Table 1 Demographic, clinical and neuropsychological features of patients 1 month before surgery and 1 year after

\begin{tabular}{|c|c|c|c|}
\hline & Pre-operative $(n=42)$ & 1 year $(n=42)$ & $p$ value \\
\hline$\overline{\text { Age }}$ & $64[45-70]$ & - & - \\
\hline Gender (male/female) & $26 / 16$ & - & - \\
\hline Disease duration & $10[6-17]$ & - & - \\
\hline Educational level & $11[8-22]$ & - & - \\
\hline LEDD (mg/day) & 1388 [187-2630] & 887.5 [350-2500] & $<0.001$ \\
\hline UPDRS I & $1[0-7]$ & $1[0-6]$ & 0.64 \\
\hline Intellectual impairment & $0[0-1]$ & $0[0-2]$ & 0.75 \\
\hline Thought disorder & $0[0-2]$ & $0[0-1]$ & 0.16 \\
\hline Depression & $1[0-3]$ & $0[0-3]$ & 0.59 \\
\hline Motivation & $0[0-4]$ & $0[0-3]$ & 0.26 \\
\hline \multicolumn{4}{|l|}{ UPDRS II } \\
\hline OFF drug (and OFF stim) & $16[6-32]$ & $14[5-41]$ & 0.59 \\
\hline ON drug (and ON stim) & $6[0-20]$ & $8[1-26]$ & $<0.001$ \\
\hline \multicolumn{4}{|l|}{ UPDRS III } \\
\hline \multicolumn{4}{|l|}{ Pre-operative } \\
\hline OFF drug & $32[8-70]$ & & \\
\hline ON drug & $10[3-28]$ & & $<0.001^{a}$ \\
\hline $\begin{array}{l}\text { Post-operative OFF } \\
\text { drug/OFF stim }\end{array}$ & & $36[11-86]$ & \\
\hline OFF drug/ON stim & & $21[7-53]$ & $<0.001^{\mathrm{b}}$ \\
\hline ON drug/ON stim & & $13[2-41]$ & $<0.001^{c}$ \\
\hline UPDRS IV & $7[2-16]$ & $3[0-8]$ & $<0.001$ \\
\hline \multicolumn{4}{|l|}{ Hoehn and Yahr } \\
\hline OFF drug (and OFF stim) & $2.5[0-4]$ & $2.5[1-5]$ & 0.51 \\
\hline ON drug (and ON stim) & $2[0-3]$ & $2[0-4]$ & 0.11 \\
\hline \multicolumn{4}{|l|}{ Mattis DRS } \\
\hline Attention & $36[31-37]$ & $36[31-37]$ & 0.62 \\
\hline Initiation/perseveration & $34[28-37]$ & $32[25-37]$ & 0.003 \\
\hline Construction & $6[4-6]$ & $6[2-6]$ & 0.21 \\
\hline Concepts & 38 [30-39] & 38 [31-39] & 0.67 \\
\hline Memory & $24[20-25]$ & 24 [19-25] & 0.47 \\
\hline Total & 135 [129-144] & 134 [112-143] & 0.075 \\
\hline \multicolumn{4}{|l|}{ Digit-span } \\
\hline Forward & $6[4-11]$ & $6[3-8]$ & 0.42 \\
\hline Backward & $5[2-8]$ & $4[2-7]$ & 0.007 \\
\hline FABB & $16[11-18]$ & $16[12-18]$ & 0.29 \\
\hline MMSE & $28[25-30]$ & 28 [21-29] & 0.002 \\
\hline
\end{tabular}

According to statistical distribution, values are expressed as median [range]

FABB frontal assessment battery at bedside, DRS dementia rating scale, LEDD levodopaequivalent daily dose, MMSE mini-mental state examination, UPDRS unified Parkinson's disease rating scale. a vs ON drug (pre-operative), b vs OFF drug/OFF stim (post-operative), $c$ vs OFF drug/ON stim (post-operative

Neither the demographic nor the pre-operative clinical variables were correlated with variation in the cognitive tests except for (1) the Hoehn and Yahr score with the MMSE $(r=-$ 
$0.40, p=0.009)$ and the construction subscore of the MDRS $(r=-0.37, p=0.020)$ and (2) educational level with the attention subscore of the MDRS $(r=-0.48, p=0.001)$. The postoperative decrease in medication was not correlated with variations in cognitive or UPDRS-I scores except with variations in the FABB $(r=0.47, p=0.006)$.

\section{Location of effective DBS contact}

At least one of the two effective contacts was located in the anterior/ventral quarter of the STN in $8 / 42$ patients. According to our other contact location criteria, 26/42 patients had an "in" location, 5/42 an "out" location, 3/42 a "border" location and 8/42 an "in/out" location.

We found no significant association between contact location and cognitive decline. However, patients with an "anterior" contact location had a tendency toward greater cognitive decline than for other patients, as measured with the MDRS total score $(-3.6 \pm 7.4$ vs $-1.5 \pm$ 6.3 points, $p=0.11)$ and with the initiation/perseveration subscore $(-4 \pm 2.9$ vs $-1.5 \pm 3.9$ points, $p=0.12$.

\section{Anatomical correlations of neuropsychological performances}

At baseline, in this selected population of patients with PD, the pre-operative total MDRS score was correlated with the volumes of right and left thalami $(r=0.46, p=0.002$ and $r=0.32, p=$ $0.038)$. The pre-operative FABB score was correlated with right and left superior frontal gyrus thickness $(r=0.43, p=0.006$ and $r=0.38, p=0.015)$.

We screened for pre-operative anatomical substrate that could explain variations in cognitive performance 1 year after bilateral STN-DBS. Regarding the univariate analyses, we found significant correlations between certain anatomical structures and variations of the digitspan tasks, the total MDRS score and MDRS attention, initiation/perseveration, memory and construction subscores (summarized in a color-coded heatmap in Fig. 1). The strongest correlation was found between the variation in the MDRS initiation/perseveration subscore and the pre-operative volume of the left nucleus accumbens $(r=0.56, p<0.001$, Bonferroniadjusted significance threshold of $p<0.0014$ ). No significant correlation was found between brain structures assessed before surgery and variations in the MMSE and FABB.

Regarding the multivariate analyses, after adjustment for age, gender, disease severity at baseline (Hoehn and Yahr, off treatment), decrease in LEDD after surgery and "anterior" contact location, correlations were significant between (1) the variation of total MDRS and the left lateral ventricle volume $(\beta=-5.73, p=0.032)$, $(2)$ between the variation of the initiation/perseveration subscore and the left nucleus accumbens volume $(\beta=1400.6, p=$ $0.042)$ and the left lateral ventricle volume $(\beta=-13.21, p=0.017)$ and (3) between the variation of the backward digit-span task and the right and left superior frontal gyrus thickness ( $\beta=3.12, p=0.004$ and $\beta=2.99, p=0.007$ respectively). In these regression models, MRI measures increased the proportion of the variance of cognitive decline that could be explained by baseline factors. For instance, age, gender, disease severity, decrease in LEDD after surgery and "anterior" contact location predicted only $21.2 \%$ of the variance of the initiation/perseveration subscore decline, but the explained variance increased to $34.4 \%$ when the volume of the nucleus accumbens was added into the statistical model.

Interestingly, the left nucleus accumbens volume was correlated with the right and left lateral ventricle volumes $(r=-0.42, p=0.003$ and $r=-0,37, p=0.015$, respectively) and (as a trend) with the left orbitofrontal cortex thickness $(r=0.30, p=0.057)$. 
Fig. 1 Heatmap representation of correlation coefficients between pre-operative MRI variables and the variation of cognitive scores 1-year after DBS-STN. MRI variables represent deep gray matter volumes (normalized on intracranial cavity volume) or cortical thickness. Correlation coefficients (Spearman $r$ ) are color-coded as shown on the top of the figure. Since lateral ventricle volumes were the only parameter negatively correlated with cognitive decline (expansion vs atrophy), the absolute values of correlation coefficient $(|r|)$ are represented here to simplify the figure. ant anterior, $G$ gyrus, inf inferior, $L$ left, lat lateral, mid middle, orbito orbitofrontal, post posterior, $\mathrm{R}$ right, sup superior

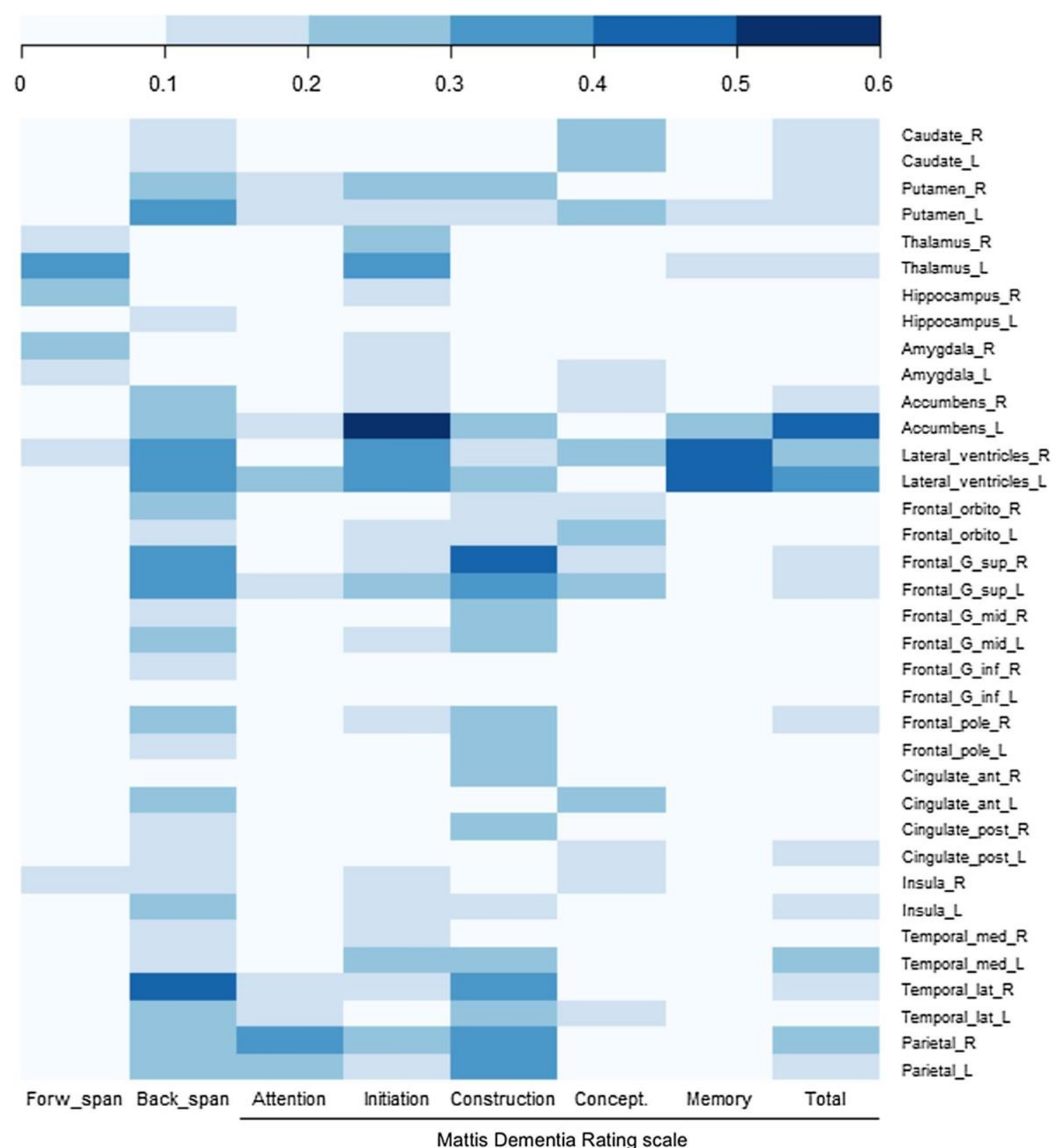

MRI measures in patients with cognitive decline

For each cognitive score, we split the patients into two distinct groups. One group was composed of patients with at least a slight decline $(\mathrm{d}<-0.2)$ compared to their own preoperative scores and the second group was composed of all the other patients. We then compared the pre-operative MRI measures of the relevant anatomical structures found in the correlation analyses between the two groups. The strength of brain atrophy/expansion was calculated with Cohen's $d$ and summarized in Fig. 2. Pre-operative left nucleus accumbens 
atrophy was associated with post-operative initiation/perseveration decline with the largest effect size $(\mathrm{d}=-1.64,95 \% \mathrm{Cl}-2.35$ to -0.91$)$. Furthermore, this was the only remaining statistically significant association $(p=0.05)$ in the multivariate analyses, after adjustment for age, gender, disease severity, decrease in LEDD after surgery, and "anterior" contact location.

Fig. 2 Forest plot representation of the effect size (Cohen's d) of pre-operative atrophy/expansion of relevant anatomical structures between patients with cognitive decline and cognitively stable patients 1-year after DBS-STN. Plots represent Cohen's d value for each association and lines represent $95 \%$ confidence interval

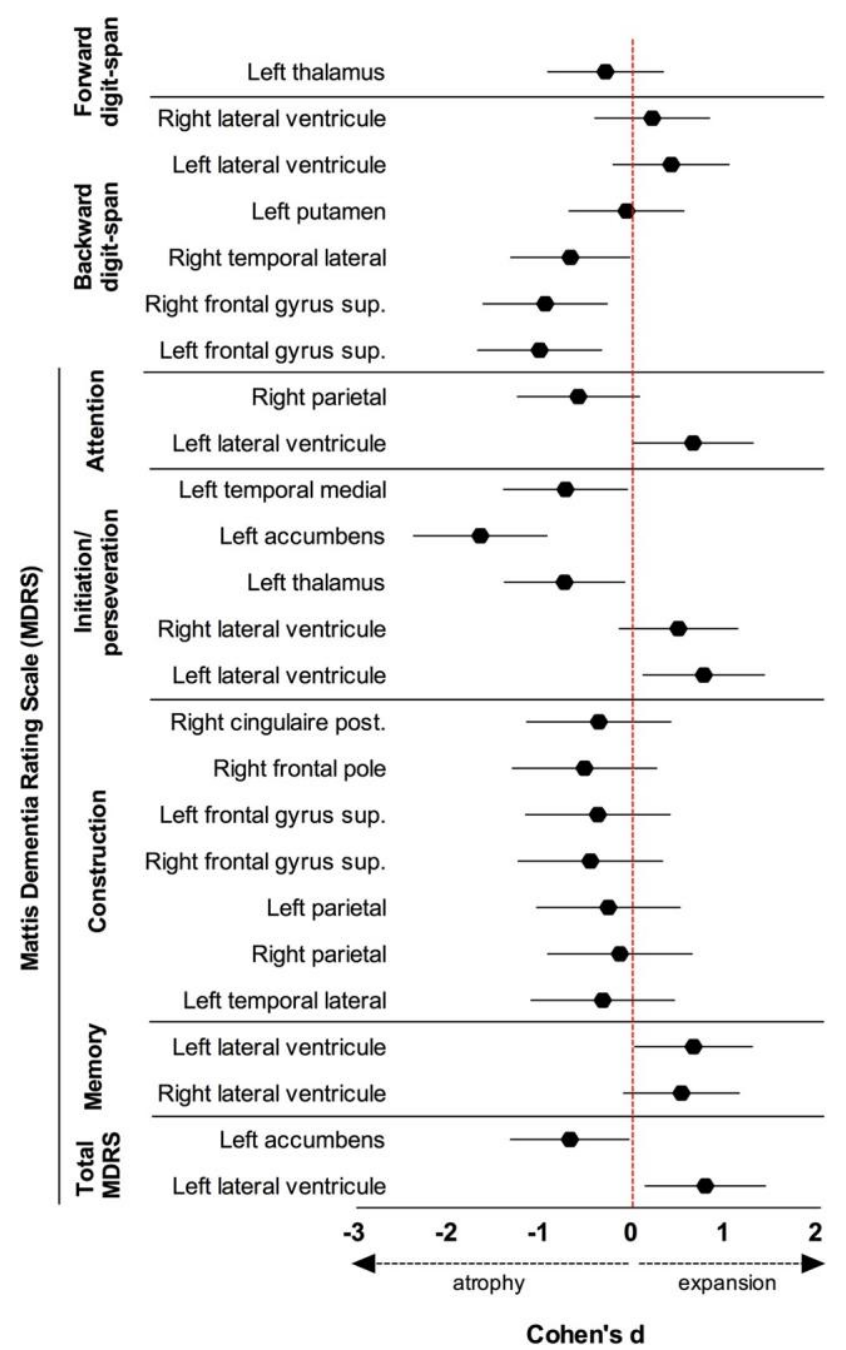

Prediction of post-operative cognitive decline

Regarding the strong association between left nucleus accumbens atrophy and decline in initiation/perseveration performances, we tested whether this pre-operative volumetric parameter could discriminate patients who will experience cognitive decline in this MDRS subscore 1-year after DBSSTN from those who will remain stable. Using ROC analysis, we showed that a threshold value of (normalized) left nucleus accumbens volume $(0.2296 \%$ of 
intracranial cavity volume) discriminated stable patients $(n=15 / 42)$ from those with future initiation/perseveration decline $(n=27 / 42)$ with $96.3 \%$ sensitivity, $80.0 \%$ specificity, $92.9 \%$ positive predictive value (PPV) and 92.9\% negative predictive value (NPV) (area under the curve $(A \cup C)=0.92, p<0.001$; Fig. 3 )

Fig. 3 Pre-operative left nucleus accumbens volume predicts cognitive decline in executive functions 1-year after DBS-STN. a ROC curve defined a threshold volume (expressed as a percentage of intracranial cavity volume) that discriminates patients who will experience cognitive decline from patients who will remain stable in the initiation/perseveration subscore of the MDRS 1-year after surgery with $96.3 \%$ sensitivity, $80.0 \%$ specificity, $92.9 \%$ positive predictive value and $92.9 \%$ negative predictive value. b Plotted left nucleus accumbens volumes in both patients with post-operative cognitive decline and cognitively stable patients. AUC area under the curve, ICC intracranial cavity

A

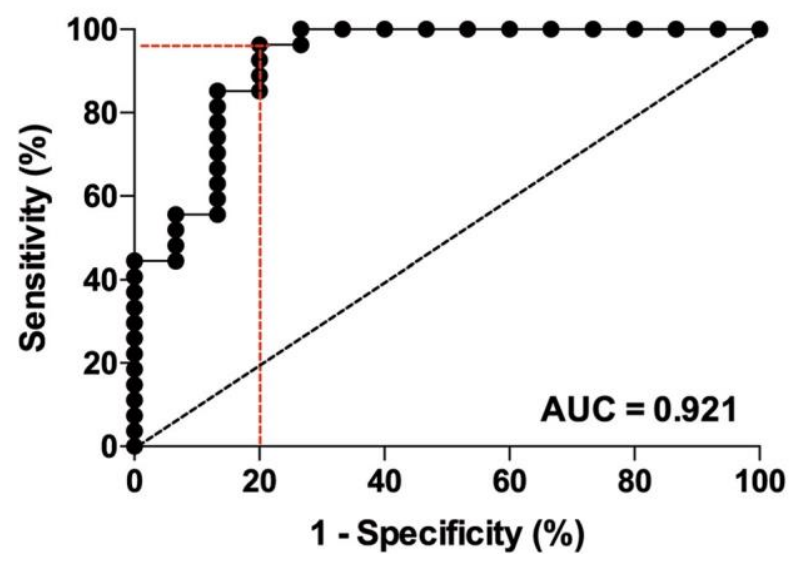

B

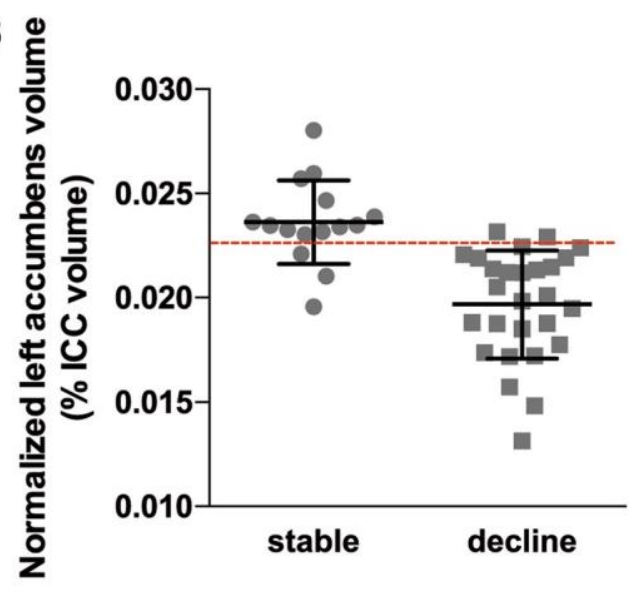

For the sensitivity analysis, we changed our cut-off to define cognitive decline after bilateral STN-DBS. Then, we tried to discriminate patients with a more severe criterion of - 1SD (i.e., $\sim d \leq-0.5)$ compared to their pre-operative scores $(n=16 / 42)$ compared to all the others $(n$ $=26 / 42$ ). In this condition, the left nucleus accumbens volume still remained a statistically significant predictor of (more severe) initiation/perseveration decline but with poorer accuracy (AUC $=0.72, p=0.016,87.5 \%$ sensitivity, $57.7 \%$ specificity, $54.0 \%$ PPV and $83.3 \%$ NPV).

\section{Discussion}

In this study, not only the left nucleus accumbens and the left lateral ventricle volumes but also the right and left superior frontal gyrus thickness measured on the pre-operative brain MRI appear to be correlated with cognitive decline after bilateral STN-DBS in patients with PD, independently of age, gender, disease severity, medication change and anterior/ventral contact locations. Furthermore, the pre-operative left nucleus accumbens volume predicts cognitive decline on executive functions (the initiation/perseveration subscore of the MDRS, mainly driven by verbal fluency performances) with good accuracy.

Despite the strict selection criteria of our cohort of patients with advanced PD undergoing bilateral STN-DBS, we observed cognitive decline 1-year after surgery. Cognitive decline was mainly observed in executive functions and especially not only in the initiation/perseveration 
subscore of MDRS with a medium effect size, but also in attention/working memory performances with a small effect size, which is consistent with data from clinical trials (Witt et al. 2008). The clinical characteristics at baseline failed to explain this outcome. Although it does not reach statistical significance, the anterior location of contacts within the STN tended to be associated with cognitive decline, as described previously (Welter et al. 2014; Tsai et al. 2007). The ability of MRI to predict cognitive decline exceeds these usual parameters and it increases the proportion of the variance of cognitive decline that could be explained by baseline factors. Thus, as well as clinical or demographical factors, volumetric MRI could become a relevant clinical tool for selecting patients before surgery.

Our results are consistent with previous MRI studies investigating cognitive impairment in patients with PD outside the field of DBS. In particular, atrophy of the left nucleus accumbens and frontal cortical thinning have been associated with mild cognitive impairment (MCl) in patients with PD (Mak et al. 2015; Ibarretxe-Bilbao et al. 2012) and ventricular dilatation has been correlated with evolution to dementia (Camicioli et al. 2011), probably reflecting atrophy both in cortical and subcortical areas relevant for cognition. Left nucleus accumbens atrophy has also been associated with apathy in patients with PD (Carriere et al. 2014). Some authors argue that emotion and motivation can modulate executive functions (Pessoa 2009) and consider the nucleus accumbens as a node between the executive control network and the reward network, regarding its projection to the frontal cortex and the limbic pathway. Apathy and depression did not seem to drive executive dysfunction in our cohort of patients because (1) we did not find any change regarding these symptoms in the UPDRS-I score after bilateral STN-DBS, (2) they were not correlated with post-operative cognitive decline and (3) our results were adjusted on dopatherapy changes after surgery. In addition, previous studies gave controversial results regarding the association between postoperative apathy and cognitive decline (Witt et al. 2008; Funkiewiez et al. 2004; Houvenaghel et al. 2015). However, it seems that left nucleus accumbens atrophy is much more a "marker" of global dysfunction in the mesocorticolimbic pathway rather than the real cause of cognitive decline. This hypothesis is supported by the trend of correlation between left nucleus accumbens atrophy and the thinning of the left orbitofrontal cortex, two major structures of the frontostriatal loop implicated in executive functions and working memory.

Up to now, pre-operative MRI in patients with PD has only been used to rule out severe brain abnormalities which could contraindicate surgery and/or imaging findings for another neurological disease (Lang et al. 2006). To our knowledge, the present study is the first to report pre-operative MRI predictors of cognitive decline after STN-DBS. Indeed, previous works failed to reach this aim but they were small sample size studies and used manual segmentation techniques or automated segmentation techniques restricted to few brain regions with less precise MRI sequences (Bonneville et al. 2005; Markser et al. 2015). One study associated pre-operative hippocampal atrophy with conversion to dementia after STNDBS, but these results were not adjusted for usual confounding factors and the authors failed to identify a predictive cut-off volume due to measurement heterogeneity (Aybek et al. 2009).

From the pathophysiological point of view, a limitation of this study is the lack of a matched control group (without DBS) to assess if patients with nucleus accumbens atrophy would also experience cognitive decline independently of surgery. However, such a controlled study would be considered non-ethical because all patients should benefit from surgery if there is no contra-indication. Furthermore, we already know from previous randomized controlled trials that the decrease in verbal fluency after STN-DBS is due to the surgery itself and not to the progression of the disease (Witt et al. 2008). Thus, it seems that the interaction between bilateral STN-DBS and brain atrophy in relevant anatomical structures leads to executive dysfunction in patients with PD. The lack of cognitive decline after STN-DBS in patients with 
early motor fluctuations (Schuepbach et al. 2013), likely having less brain atrophy, corroborates this hypothesis.

Another limitation of this study is its relative small sample size and our findings on the predictive value of brain MRI for cognitive decline after STN-DBS must be validated in other cohorts. Our results must also be confirmed after a longer follow-up period because we can postulate that the small or medium effect-size of cognitive decline observed here 1 year after STN-DBS will lead to more global and more severe cognitive impairment years afterwards. Indeed, several studies have demonstrated that patients with PD-MCl or with slight dysexecutive syndrome had a high risk of developing dementia (Pigott et al. 2015), although these conclusions are less affirmative for selected patients that underwent STN-DBS (Krack et al. 2003; Contarino et al. 2007). We must also acknowledge that we used global cognitive scales such as MDRS, FABB, MMSE and digit span tasks. Thus, it would be interesting in future studies to use a larger cognitive battery and also specific apathy, anxiety and depression scales, to evaluate more precisely each cognitive domain and its putative psychiatric correlates. Finally, we can question whether left nucleus accumbens atrophy is really a predictor of a clinically meaningful cognitive decline. Indeed, post-operative dementia is rare after STN-DBS and the present study was only designed to assess smallto-medium cognitive decline. However, small-to-medium postsurgical decline could propel patients from borderline impairment into the moderate-to-severe range of cognitive impairment. Furthermore, even if we consider more severe cognitive impairment (-1SD from baseline), left nucleus accumbens atrophy remained a statistically significant predictor of cognitive decline in the initiation/perseveration subscore, demonstrating its clinical relevance.

\section{Conclusion}

Our study offers a new marker to predict a decrease in executive functions after bilateral STNDBS in patients with PD, in addition to conventional selection criteria and unpredictable final contact location. Now that normative and fully automated brain segmentation software applications are freely available, this work also offers new clinical perspectives for identifying more suitable candidates for STN-DBS.

Acknowledgements The authors want to thank Christine Delaigue, coordinating nurse for the Parkinson's Disease Center in Clermont-Ferrand University Hospital. They also thank Prof. Thomas Tourdias and Prof. Vincent Dousset from Bordeaux University Hospital for making available their MRI analysis facilities, and Celine Lambert from Clermont-Ferrand University Hospital for her technical assistance.

Author contributions Conception of the project: VP and FD. Organization: FD. Execution: all authors. Statistical Analysis: VP and BP. Writing of the first draft: VP. Review and Critique: all authors. Study concept and design: VP, BP, JJL and FD.

Funding Hospital Program for Clinical Research at Clermont-Ferrand University Hospital.

\section{Compliance with ethical standards}

Conflict of interest VP received travel expenses and/or consulting fees from the ARSEP Foundation, Biogen, Teva-Lundbeck and Merk-Serono, unrelated to the submitted work. FD serves on scientific advisory boards and has received honoraria and research support for his institution from Novartis, Teva-Lundbeck, Allergan, Aguettant, Servier and Merz, unrelated to the submitted work. Other authors: no conflict of interests. 


\section{References}

Alegret M, Junqué C, Valldeoriola F et al (2001) Effects of bilateral subthalamic stimulation on cognitive function in Parkinson disease. Arch Neurol 58:1223-1227

Altman DG (1990) Practical statistics for medical research. CRC Press,Boca Raton

Aybek S, Lazeyras F, Gronchi-Perrin A et al (2009) Hippocampal atrophy predicts conversion to dementia after STN-DBS in Parkinson's disease. Parkinsonism Relat Disord 15:521-524

Bonneville F, Welter ML, Elie C et al (2005) Parkinson disease, brain volumes, and subthalamic nucleus stimulation. Neurology 64:1598-1604

Camicioli R, Sabino J, Gee M et al (2011) Ventricular dilatation andbrain atrophy in patients with Parkinson's disease with incipient dementia. Mov Disord 26:1443-1450

Carriere N, Besson P, Dujardin K et al (2014) Apathy in Parkinson's disease is associated with nucleus accumbens atrophy: a magnetic resonance imaging shape analysis. Mov Disord 29:897-903

Charles PD, Van Blercom N, Krack P et al (2002) Predictors of effective bilateral subthalamic nucleus stimulation for PD. Neurology 59:932-934

Contarino MF, Daniele A, Sibilia AH et al (2007) Cognitive outcome 5 years after bilateral chronic stimulation of subthalamic nucleus in patients with Parkinson's disease. J Neurol NeurosurgPsychiatry 78:248-252

Daniels C, Krack P, Volkmann J et al (2010) Risk factors for executive dysfunction after subthalamic nucleus stimulation in Parkinson's disease. Mov Disord 25:1583-1589

de Chazeron I, Pereira B, Chereau-Boudet I et al (2016) Impact of localisation of deep brain stimulation electrodes on motor and neurobehavioural outcomes in Parkinson's disease. J Neurol Neurosurg Psychiatry 87:758-766

Desikan RS, Ségonne F, Fischl B et al (2006) An automated labeling system for subdividing the human cerebral cortex on MRI scans into gyral based regions of interest. NeuroImage 31:968-980

Dujardin K, Devos D, Duhem S et al (2006) Utility of the Mattis dementia rating scale to assess the efficacy of rivastigmine in dementia associated with Parkinson's disease. J Neurol253:1154-1159

Emre M (2004) Dementia in Parkinson's disease: cause and treatment. Curr Opin Neurol 17:399-404

Fischl B, Dale AM (2000) Measuring the thickness of the human cerebral cortex from magnetic resonance images. Proc Natl Acad Sci USA 97:11050-11055

Funkiewiez A, Ardouin C, Caputo E et al (2004) Long term effects of bilateral subthalamic nucleus stimulation on cognitive function,mood, and behaviour in Parkinson's disease. J Neurol NeurosurgPsychiatry 75:834-839

Hanganu A, Bedetti C, Degroot C et al (2014) Mild cognitive impairment is linked with faster rate of cortical thinning in patients with Parkinson's disease longitudinally. Brain 137:1120-1129

Houvenaghel J-F, Le Jeune F, Dondaine T et al (2015) Reduced verbal fluency following subthalamic deep brain stimulation: a frontal-related cognitive defcit? PloS One 10:e0140083

Hughes AJ, Daniel SE, Kilford L, Lees AJ (1992) Accuracy of clinical diagnosis of idiopathic Parkinson's disease: a clinico pathological study of 100 cases. J Neurol Neurosurg Psychiatry55:181-184

Ibarretxe-Bilbao N, Junque C, Segura B et al (2012) Progression of cortical thinning in early Parkinson's disease. Mov Disord 27:1746-1753

Kim H-J, Jeon BS, Paek SH et al (2014) Long-term cognitive outcome of bilateral subthalamic deep brain stimulation in Parkinson's disease. J Neurol 261:1090-1096

Krack P, Batir A, Van Blercom N et al (2003) Five-year follow-up of bilateral stimulation of the subthalamic nucleus in advanced Parkinson's disease. N Engl J Med 349:1925-1934

Lang AE, Houeto J-L, Krack P et al (2006) Deep brain stimulation: preoperative issues. Mov Disord 21(Suppl 14):S171-S196

Le Goff F, Derrey S, Lefaucheur R et al (2015) Decline in verbal fluency after subthalamic nucleus deep brain stimulation in Parkinson's disease: a microlesion effect of the electrode trajectory? J Park Dis 5:95-104 
Lemaire J-J, Coste J, Ouchchane L et al (2007) Brain mapping in stereotactic surgery: a brief overview from the probabilistic targeting to the patient-based anatomic mapping. NeuroImage 37(Suppl 1):S109-115

Lemaire J-J, Pereira B, Derost P et al (2016) Subthalamus stimulation in Parkinson disease: accounting for the bilaterality ofcontacts. Surg Neurol Int 7:S837-S847

Mak E, Su L, Williams GB et al (2015) Baseline and longitudinal grey matter changes in newly diagnosed Parkinson's disease: ICICLE-PD study. Brain 138:2974-2986

Manjón JV, Coupé P (2016) vol Brain: an online MRI brain volumetry system. Front Neuroinform 10:30

Markser A, Maier F, Lewis CJ et al (2015) Deep brain stimulation and cognitive decline in Parkinson's disease: the predictive value of electroencephalography. J Neurol 262:2275-2284. d

Marson DC, Dymek MP, Duke LW, Harrell LE (1997) Subscale validity of the Mattis dementia rating scale. Arch Clin Neuropsychol 12:269-275

Næss-Schmidt E, Tietze A, Blicher JU et al (2016) Automatic thalamus and hippocampus segmentation from MP2RAGE: comparison of publicly available methods and implications for DTI quantifcation. Int J Comput Assist Radiol Surg 11:1979_ 1991

Parsons TD, Rogers SA, Braaten AJ et al (2006) Cognitive sequelae of subthalamic nucleus deep brain stimulation in Parkinson's disease:a meta-analysis. Lancet Neurol 5:578-588

Pessoa L (2009) How do emotion and motivation direct executive control? Trends Cognit Sci 13:160-166

Pigott K, Rick J, Xie SX et al (2015) Longitudinal study of normal cognition in Parkinson disease. Neurology 85:1276-1282

Saint-Cyr JA, Trépanier LL, Kumar R et al (2000) Neuropsychological consequences of chronic bilateral stimulation of the subthalamic nucleus in Parkinson's disease. Brain 123(Pt 10):2091-2108

Schuepbach WMM, Rau J, Knudsen K et al (2013) Neurostimulation for Parkinson's disease with early motor complications. N Engl J Med 368:610-622

Segura B, Baggio HC, Marti MJ et al (2014) Cortical thinning associated with mild cognitive impairment in Parkinson's disease. MovDisord 29:1495-1503

Smeding HMM, Speelman JD, Huizenga HM et al (2011) Predictors of cognitive and psychosocial outcome after STN DBS in Parkinson's Disease. J Neurol Neurosurg Psychiatry 82:754-760

Thobois S (2006) Proposed dose equivalence for rapid switch between dopamine receptor agonists in Parkinson's disease: a review of the literature. Clin Ther 28:1-12

Tsai S-T, Lin S-H, Lin S-Z et al (2007) Neuropsychological effects after chronic subthalamic stimulation and the topography of the nucleus in Parkinson's disease. Neurosurgery 61:E1024-E1029(discussion E1029-E1030).

Welter ML, Houeto JL, Tezenas du Montcel S et al (2002) Clinical predictive factors of subthalamic stimulation in Parkinson's disease. Brain 125:575-583

Welter M-L, Schüpbach M, Czernecki V et al (2014) Optimal target localization for subthalamic stimulation in patients with Parkinson disease. Neurology 82:1352-1361

Witt K, Daniels C, Reiff J et al (2008) Neuropsychological and psychiatric changes after deep brain stimulation for Parkinson's disease:a randomised, multicentre study. Lancet Neurol 7:605-614

Witt K, Granert O, Daniels C et al (2013) Relation of lead trajectory and electrode position to neuropsychological outcomes of subthalamic neurostimulation in Parkinson's disease: results from a randomized trial. Brain 136:2109-211 